\title{
Efficient translocation of positively charged residues of M13 procoat protein across the membrane excludes electrophoresis as the primary force for membrane insertion
}

\author{
Andreas Kuhn, Heng-Yi Zhu' ${ }^{1}$ and \\ Ross E.Dalbey ${ }^{1}$
}

Department of Microbiology, Biozentrum, University of Basel, Klingelbergstrasse 70, CH-4056 Basel, Switzerland and ${ }^{1}$ Department of Chemistry, Ohio State University, Columbus, OH 43210, USA

Communicated by G.Kreil

The coat protein of bacteriophage M13 is inserted into the Escherichia coli plasma membrane as a precursor protein, termed procoat, with a typical leader peptide of 23 amino acid residues. Its membrane insertion requires the electrochemical potential but not the cellular components SecA and SecY. Since the electrochemical gradients result in the periplasmic side of the membrane being positively charged, the membrane potential could contribute to the transfer of the negatively charged central region of procoat across the membrane. Here we demonstrate that the central domain following the leader peptide can be translocated across the membrane even when the net charge of the region is changed from -3 to +3 . This rules out an electrophoresis-like insertion mechanism for procoat. We also show that the sec independence of procoat insertion is linked to the presence of the second apolar domain. The deletion of most of the second apolar domain from a procoat fusion protein results in sec dependent membrane insertion of the hybrid protein. Moreover, like other proteins that require the sec genes, translocation of this sec dependent procoat protein is inhibited when positively charged residues are introduced after the leader peptide. Loop models involving one or two hydrophobic regions are presented that account for the differences in tolerance of positively charged residues. Key words: membrane insertion/M13 coat protein/sec dependence

\section{Introduction}

While certain bacterial membrane proteins can insert directly into the membrane, most exported or membrane proteins use an insertion mechanism involving the sec gene products (Wickner, 1989). During, or shortly after their synthesis, some exported proteins first interact with cytoplasmic proteins, termed molecular chaperonins. This interaction maintains the exported protein in a loose conformation that is competent for membrane insertion (Randall and Hardy, 1986). The chaperonin exported protein complex is then thought to bind to the membrane associated SecA protein, where it is transferred to the SecY component under ATP consumption. The detailed mechanism by which the protein is actually translocated through the membrane still has to be elucidated at its molecular level.

The bacterial proteins that directly insert into the membrane include primarily small proteins such as the procoat protein of bacteriophage M13 (Wolfe et al., 1985; Cobet et al., 1989). The membrane insertion of the procoat protein has been extensively studied and it has been found that (i) procoat binds to the membrane via electrostatic interactions between the positively charged terminal regions of the protein and the acidic phospholipid head groups (Gallusser and Kuhn, 1990); (ii) procoat insertion occurs by the formation of a transmembrane loop (Kuhn, 1987) which requires the electrochemical membrane potential (Date et al., 1980); (iii) procoat is cleaved to coat by leader peptidase after membrane insertion (Kuhn and Wickner, 1985). Despite numerous studies, the precise role of the electrochemical potential in transferring the acidic central region of procoat has remained elusive. One possible mechanism is an electrophoresis-like transfer of the negatively charged residues of the extracellular region of procoat, since due to the potential the outer face of the membrane is positively charged.

In the present study we investigated the role in protein insertion of the acidic residues of procoat at positions +2 , +4 and +5 . These residues were substituted with neutral or positively charged amino acids using site-directed mutagenesis. Surprisingly, when all three residues were replaced with positively charged arginines, the membrane insertion of procoat still occurred, although at a slower rate. This result is fundamentally different from those of other exported proteins, where a single positively charged residue inhibits membrane insertion (Li et al., 1988; Yamane and Mizushima, 1988). The different effects of positive charges on the export of these proteins will be discussed in terms of the Sec machinery and loop models for protein translocation.

\section{Results}

The insertion of $M 13$ procoat across the membrane does not require an acidic central region

To investigate whether the negatively charged residues in the translocated region of the M13 procoat protein have an essential function for membrane transfer, we generated a number of site-directed mutations in the region following the leader peptide. Figure 1A shows the mutations that were created and summarizes the translocation properties of the mutants, which were named according to the sequence of the first five amino acids of the mature region. Mutants were examined by pulse-labeling cells with $\left[{ }^{35} \mathrm{~S}\right]$ methionine, and analyzed by SDS-PAGE and fluorography. Wild-type procoat (Figure 1B, lane 1) and most of the mutants (lanes $2-7$ ), which had been labeled during a $1 \mathrm{~min}$ pulse, were almost completely processed to coat protein during this time. A subtle retardation of processing was observed when a tryptophan was introduced at position +4 (lanes 4 and 5), but processing was even faster than wild-type when two asparagines replaced the aspartic acid residues at +4 and +5 (lane 6). Therefore, the reduction of the net charge from 


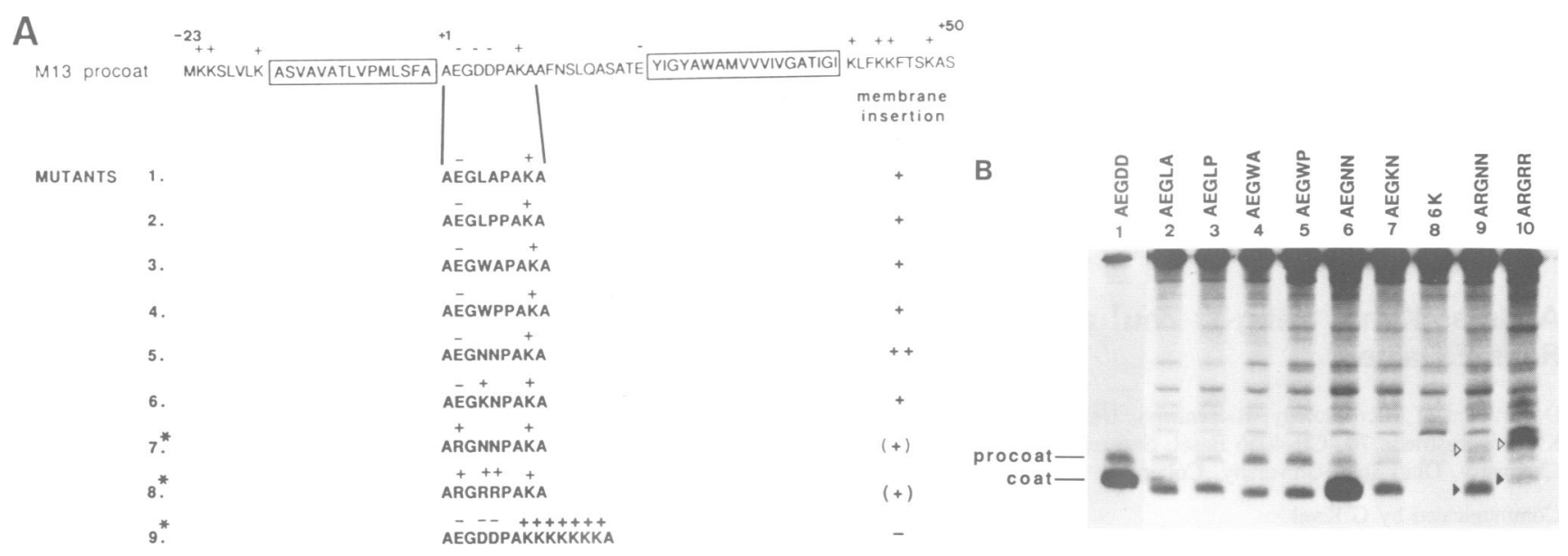

Fig. 1. (A) M13 procoat mutants and their membrane insertion properties. Substitutions $(1-8)$ were made at positions $+2,+4$ and +5 in the central region of procoat. Procoat $6 \mathrm{~K}$ has six lysines (9) inserted between residues +8 and +9 . The asterisk marks the mutants that were studied with fusion proteins as well. These proteins were made by C-terminal extension of procoat with 103 amino acids derived from leader peptidase (lep). This modification of procoat does not interfere with its membrane insertion (Kuhn et al., 1986a, 1987). (B) Processing of procoat mutants. E.coli HJM114 containing wild-type or mutant plasmids was grown to the mid-log phase in M9 medium containing $0.5 \%$ fructose supplemented with 19 amino acids (without methionine) at $37^{\circ} \mathrm{C}$. After induction of the cells with IPTG $(1 \mathrm{mM})$ for $15 \mathrm{~min}$, the cells were incubated with $\left[{ }^{35} \mathrm{~S}\right] \mathrm{methionine}$ for $1 \mathrm{~min}$. The cells were then acid-precipitated and immunoprecipitated with antisera to coat protein. The samples were then analyzed by SDS-PAGE and fluorography. Procoat $6 \mathrm{~K}$ (lane 8 ) is not recognized by the antibody. The arrowheads in lanes 9 and 10 correspond to procoat ( $\downarrow$ ) and coat protein $(\triangleright)$.

-2 to 0 in the region following the leader peptide has no inhibitory effect on membrane translocation of M13 procoat. Moreover, even increasing the net charge to +2 by arginyl substitution (lane 9), did not severely affect insertion kinetics.

\section{Positively charged residues in the translocated region gradually slow down membrane translocation}

Further increases in the net charge of the central region to +3 (lane 10) slowed the processing of procoat. Approximately $20 \%$ of procoat ARGRR was converted to the coat protein in a $1 \mathrm{~min}$ labeling. These results with procoat are in sharp contrast to those found with other proteins, where the introduction of a single arginine residue has a severe inhibitory effect on translocation (Li et al., 1988; Yamane and Mizushima, 1988).

To quantify the extent of processing better and to avoid interference of the mutations with the antigenic recognition, we fused procoat at the C-terminal alanine to a 103 amino acid-long antigenic fragment of leader peptidase (lep). Previously, we have shown that the membrane insertion of the wild-type procoat fused to this fragment has normal kinetics and that the leader peptidase fragment remains on the cytoplasmic surface of the membrane (Kuhn et al., 1986a). Wild-type and mutant procoat-lep fusions were pulse-labeled for $20 \mathrm{~s}$ and chased for either $5 \mathrm{~s}$ or $5 \mathrm{~min}$ (Figure 2). For the wild-type protein (procoat-lep AEGDD), the uncleaved form is not detectable at the earliest chase point (lane 1), indicating that it rapidly assembles across the membrane. The mutant procoat-lep ARGNN was processed at a slightly slower rate and showed $\sim 20 \%$ unprocessed fusion protein at the $5 \mathrm{~s}$ chase (compare lanes 3 and 4). An even more pronounced retardation in processing was observed with the mutant ARGRR, showing 50\% unprocessed form immediately following the pulse-labeling (lanes 5 and 6). However, the introduction of six additional lysinyl residues (procoat $6 \mathrm{~K}-\mathrm{lep}$ ) completely inhibited the processing (lanes 7 and 8).

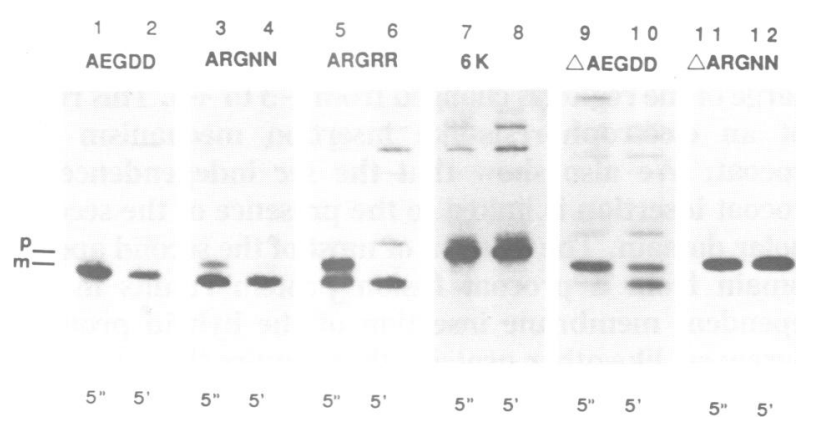

Fig. 2. Positively charged amino acids gradually inhibit membrane insertion. Procoat-lep fusion proteins with either the wild-type procoat sequence (lanes 1 and 2), ARGNN (lanes 3 and 4), ARGRR (lanes 5 and 6) or the $6 \mathrm{~K}$ sequence (lanes 7 and 8) were expressed in E.coli LC137. The corresponding fusion proteins with either wild-type sequence (lanes 9 and 10) or ARGNN (lanes 11 and 12), both of which have procoat residues +27 to +48 deleted, were also studied. The $\mathrm{p}$ and $\mathrm{m}$ correspond to the precursor and mature form, respectively.

\section{Positively charged residues are only translocated across the membrane when both hydrophobic regions of $M 13$ procoat are present}

To investigate whether the M13 procoat tolerates the four positively charged residues because its two hydrophobic regions favor the formation of a transmembrane loop, we deleted most of the second hydrophobic region (residues +27 to +48 ). As shown in Figure 2, this protein ( $\Delta$ procoat - lep AEGDD) inserts across the membrane, though at a reduced rate (lanes 9 and 10). However, the introduction of one positively charged residue into this protein $(\Delta$ procoat - lep ARGNN) totally inhibited processing to the mature form (lanes 11 and 12).

The location of precursor and mature forms within the cell was analyzed by proteinase $\mathrm{K}$ accessibility (Figure 3 ). The cells were pulse-labeled with $\left[{ }^{35}\right.$ S $]$ methionine, the outer membrane was permeabilized with sucrose, Tris and EDTA 
and digested with $1 \mathrm{mg} / \mathrm{ml}$ proteinase $\mathrm{K}$ for $60 \mathrm{~min}$. The precursor forms of the mutants including procoat $6 \mathrm{~K}-$ lep (lanes 4 and 5) and $\Delta$ procoat-lep ARGNN (lanes 10 and 11) were resistant to the proteinase, confirming that membrane translocation is the retarded step. For procoat-lep ARGRR (lanes 1-3) the mature form was clipped by the protease to a slightly shorter fragment, indicating that the C-terminal part encompassing the antigenic region was located in the cytoplasm. This had been observed for wildtype procoat-lep earlier (Kuhn et al., 1986a). However, $\Delta$ coat-lep AEGDD (lanes 7-9) with only one hydrophobic region was entirely digested by the protease, confirming that the antigenic region had traversed the plasma membrane. The cellular conformations of the different mutants are summarized in Figure 4.

\section{Positively charged residues inhibit membrane translocation of single-spanning, sec dependent proteins}

To test whether the second hydrophobic region is essential for the $s e c$ independent translocation of procoat-lep, the plasmid encoding deletion mutant $\Delta$ procoat-lep AEGDD was transformed into the temperature sensitive strains CJ105 $\left(\sec A^{\text {ts51 }}\right)$ and $\mathrm{CJ} 107\left(\sec Y^{\mathrm{ts} 24}\right)$. The transformants were
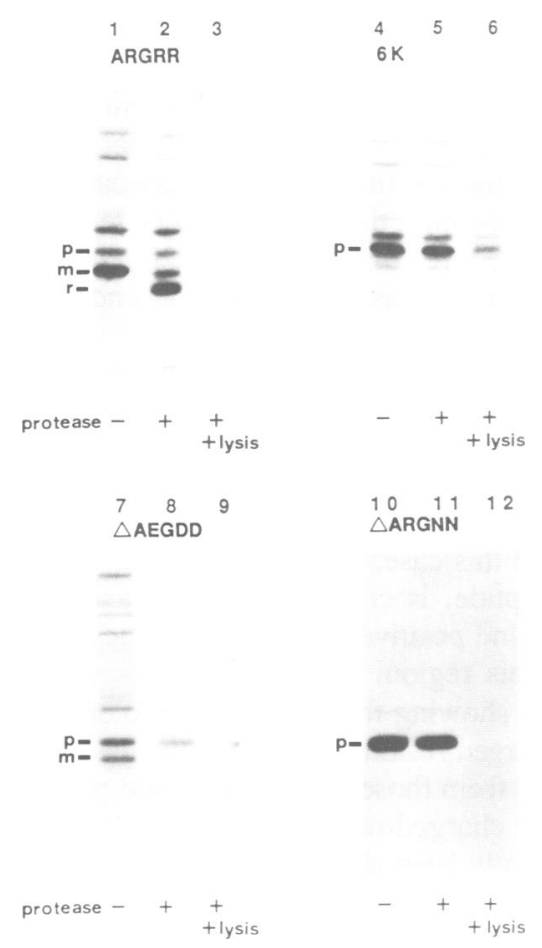

Fig. 3. Protease mapping of procoat mutants. E.coli HJM 114 synthesizing procoat-lep ARGRR (lanes 1-3), procoat-lep 6K (lanes 4-6), $\triangle 27-48$ procoat - lep (lanes 7-9) or $\triangle 27-48$ procoat - lep ARGNN (lanes 10-12), was grown in M9 medium to the exponential phase and induced with $0.4 \% \mathrm{~L}$-arabinose. The cells $(0.5 \mathrm{ml})$ were pulse-labeled with $\left[{ }^{35} \mathrm{~S}\right]$ methionine $(40 \mu \mathrm{Ci})$ for $1 \mathrm{~min}$, chased with methionine $(500 \mu \mathrm{g} / \mathrm{ml})$, and treated with $0.5 \mathrm{ml}$ of buffer A $(40 \%$ sucrose, $20 \mathrm{mM}$ EDTA and $60 \mathrm{mM}$ Tris, $\mathrm{pH} \mathrm{8.0)}$ to permeabilize the outer membrane. Samples were then incubated with or without proteinase $\mathrm{K}(1 \mathrm{mg} / \mathrm{ml})$ for $60 \mathrm{~min}$. Where indicated, a portion of cells was treated with $2 \%$ Triton $\mathrm{X}-100$ to lyse the cells prior to proteinase treatment. The samples were analyzed by acid-precipitation, immunoprecipitated with antisera to leader peptidase, and subjected to SDS-PAGE and fluorography. The $\mathrm{p}, \mathrm{m}$ and $\mathrm{r}$ correspond to the precursor, mature form, and the protease resistant fragment, respectively. grown at $30^{\circ} \mathrm{C}$ and then shifted to $42^{\circ} \mathrm{C}$ for $2 \mathrm{~h}$. Portions were pulse-labeled with $\left[{ }^{35} \mathrm{~S}\right]$ methionine and analyzed by immunoprecipitation and SDS-PAGE (Figure 5). Procoatlep ARGNN (lanes 1-3) was processed in all three strains to the mature form, whereas the OmpA protein accumulated in its precursor form in both CJ105 and CJ107. However, $\Delta$ procoat - lep AEGDD was converted to the mature form only in the HJM strain (lane 4), and not in the strains CJ105 (lane 5) and CJ107 (lane 6). We conclude that the membrane insertion pathways of procoat - lep and $\Delta$ procoat - lep differ fundamentally in respect to sec dependence.

\section{Discussion}

Electrophoretic models of protein export propose that charged domains of proteins are moved across the membrane by the electric field. The M13 procoat protein provides very useful features to test this proposal directly. First, it requires the membrane potential (Date et al., 1980). In Escherichia coli, the membrane potential is such that there is a net positive charge on its outer surface and a net negative charge on the cytoplasmic side. Second, the region of the procoat protein which is translocated across has a net charge of -3 . Third, the M13 procoat protein inserts into the membrane

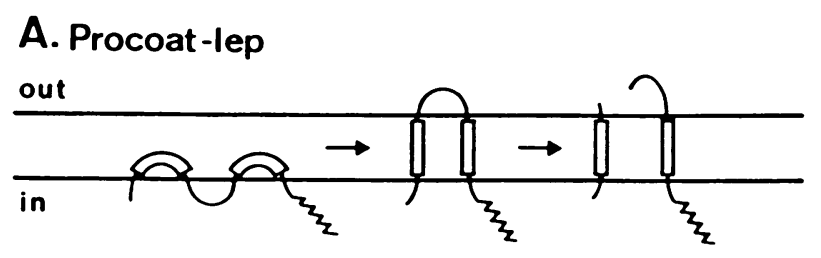

\section{B. $\triangle 27-48$ procoat-lep}

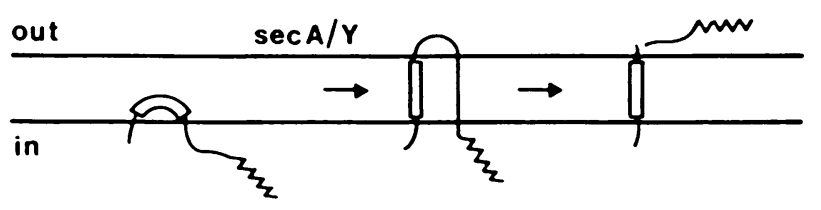

Fig. 4. Loop models of protein translocation depicting (A) the sec independent and (B) the sec dependent modes of procoat insertion. The lep region corresponds to the zig-zag line.

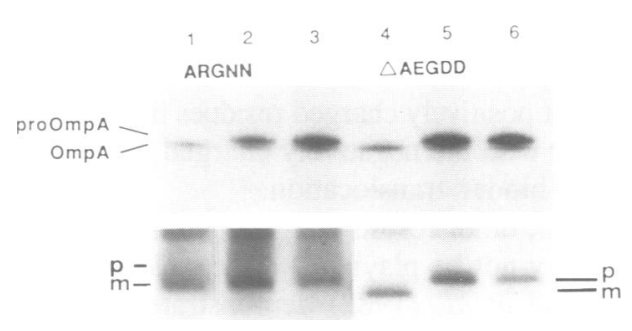

Fig. 5. Membrane insertion of $\triangle$ procoat-lep requires secA and secY. E.coli HJM 114 (lanes 1 and 4), CJ105 (lanes 2 and 5), and CJ107 (lanes 3 and 6) synthesizing procoat-lep ARGNN (lanes 1-3) or $\triangle 27-48$ procoat-lep AEGDD (lanes $4-6$ ) were grown at $30^{\circ} \mathrm{C}$ to a density of $5 \times 10^{7}$ cells $/ \mathrm{ml}$. After a temperature shift to $42^{\circ} \mathrm{C}$ for $2 \mathrm{~h}$, $0.4 \%$ arabinose was added for $30 \mathrm{~min}$. The cells were labeled with 10 $\mu \mathrm{Ci}\left[{ }^{35} \mathrm{~S}\right]$ methionine for $5 \mathrm{~min}$. The samples were acid-precipitated and immunoprecipitated with antisera to OmpA (upper panel) and to leader peptidase (lower panel), as described in Figure 1B. The $p$ and $m$ correspond to the precursor and mature forms, respectively. 
as a loop structure (Kuhn, 1987) involving both hydrophobic regions which flank the extracellular domain (Kuhn et al., 1986b). This negatively charged domain is therefore transported to the positively charged periplasmic membrane surface.

Our results reported here exclude an electrophoresis-like mechanism for the translocation of the M13 procoat protein. The procoat protein can transfer its central region across the membrane even when it has positively charged residues in the translocated region. Thus, the net charge of the procoat's central region and the electrical field do not move the protein across the membrane. Moreover, it has been shown previously that the chemical potential can substitute for the electrical component of the membrane potential (Bakker and Randall, 1984). Therefore, the requirement for an electrochemical potential during protein insertion has to have another purpose. In addition, we do not know for procoat, of course, whether the acidic and basic residues are translocated across the membrane in a truly charged or in a neutralized form surrounded by a water shell. Our results with the various procoat mutants that contain less ionic residues than the wild-type gave no clear conclusion, since some translocated faster but others slower than the wild-type protein.

Although the membrane potential is not directly involved in the insertion process, it might have an important role for the orientation of the inserting protein. It has been suggested earlier that proteins orient themselves according to the charged groups flanking the hydrophobic leader peptide region (Daniels et al., 1981; von Heijne, 1986a,b). The procoat molecule generates a favorable charge distribution even in the case of the mutant procoat (with four positively charged residues in the central region), since the cytoplasmic portions of the protein contribute seven positively charged residues.

While M13 procoat tolerates positively charged residues in the central domain, we observe a kinetic effect on membrane translocation (Figure 2). One possible reason for this is that the electric field has a minor effect on the translocation kinetics and positively charged residues slow the translocation. Another possibility is that the positively charged residues in this region interact electrostatically with the negatively charged membrane surface and hinder the translocation of this region. We favor this latter possibility since neutral residues promote faster insertion. Previously, we have shown that the M13 procoat electrostatically binds to the membrane surface by its positively charged $\mathrm{N}$ and $\mathrm{C}$ terminal regions (Gallusser and Kuhn, 1990). Thus, it is plausible that positively charged residues in the central region also interact with the negatively charged phospholipid head groups and hinder translocation.

In addition, other results suggest that the electrochemical potential may not be playing a direct role in translocation. Zimmermann et al. (1982) demonstrated that membrane insertion was dramatically less dependent on the membrane potential with a procoat mutant with a mutation at +2 . It is difficult to explain this result by an electrophoretic transfer mechanism. Possibly, the membrane potential acts indirectly, like on the packing of the phospholipids or on the precursor conformation. Furthermore, in vitro studies showed that the requirement of a membrane potential differed among various precursor proteins (Yamada et al., 1989a) and assay conditions (Yamada et al., 1989b). For example, the require- ments for a membrane potential was modulated by the amount of the SecA protein added. This suggests that the membrane potential is rather involved in the binding of the precursor to the inner surface of the membrane. M13 procoat requires for its initial binding a negatively charged inner membrane surface to which the membrane potential contributes. It is therefore plausible that a dissipation of the potential also hinders the procoat protein from binding to the membranes.

In the present study, we also tested whether the sec independent assembly of procoat was due to the second apolar domain of procoat, which participates in forming the loop structure which then inserts the central region across the lipid bilayer. Previously, we showed that procoat fused with a 103 amino acid segment derived from the polar, C-terminal domain of leader peptidase inserts into the membrane in a secY independent manner (Kuhn et al., $1986 \mathrm{~b})$. Our results here indicate that when most of the second apolar domain of procoat is deleted, then the fusion protein assembles at a reduced rate in a $\sec Y$ dependent manner. This establishes that the independence of $\sec Y$ is due to the presence of the second apolar region of procoat. However, when the two apolar regions are far apart, separated by a long stretch of $\sim 100$ amino acid residues, they lose their capacity to promote $\mathrm{sec}$ independent insertion. For example, a procoat fusion protein with 98 residues derived from the OmpA sequence inserted only in the presence of functional SecA and SecY (Kuhn, 1988). Taking these results together, we suggest that the determining factor for $\mathrm{sec}$ requirement is the size of the procoat domain to be translocated. Possibly, the Sec machinery recognizes in the preprotein some three-dimensional structure comprising the already laterally inserted leader peptide and the adjoining polar stretch within the mature domain (Figure 4B).

The sec independent protein insertion might therefore be based on a synergistic entry of both hydrophobic regions of the procoat protein into the lipid bilayer (Figure 4A). When parts of the mature hydrophobic region are deleted, the formation of a transmembrane loop has to proceed differently. In this case, the protein sequence following to the leader peptide, is critical for membrane translocation (Figure 4B), and positively charged residues are no longer tolerated in this region.

Our results showing that procoat can tolerate up to four positively charged residues following the leader peptide are very different from those of other secreted proteins, where one positively charged residue blocks membrane insertion. This has recently been shown for alkaline phosphatase $(\mathrm{Li}$ et al., 1988), lipoprotein- $\beta$-lactamase (Yamane and Mizushima, 1988) and OmpA (Zhu,H., Kuhn,A. and Dalbey,R., in preparation). It is interesting to note that exported proteins very rarely have a positive charge in the C-terminal part of the leader peptide or in the first few positions of the mature sequences (von Heijne, 1986a). Why do positively charged residues have such an inhibitory effect on all other exported proteins but not on M13 procoat? One possibility is that these positive charges prevent the proteins from interacting with the secretory apparatus (e.g. with the SecA protein). Therefore, a sec dependent procoat would be very sensitive to positively charged residues, as shown here for the sec dependent procoat mutant $\triangle \mathrm{ARGNN}$. Thus, it seems that $\mathrm{Sec}$ interactions require certain structural features of the mature sequence. 


\section{References}

Asano, T. and Hidaka,H. (1984) J. Pharmacol. Exp. Ther., 231, 141-145. Backlund,Jr,P.S., Akasamit,R.R., Unson,C.G., Goldsmith,P., Speigel,A.M. and Milligan,G. (1988) Biochemistry, 27, 2040-2046.

Becker,S., Warren,M.K. and Haskill,S. (1987) J. Immunol., 139, 3703-3709.

Berridge,M.J. (1987) Annu. Rev. Biochem., 56, 159-193.

Besterman,J.M. and Cuatrecasas,P. (1984) J. Cell Biol., 99, 340-343.

Besterman,J.M., Duronio,V. and Cuatrecasas,P. (1986) Proc. Natl. Acad. Sci. USA, 83, 6785-6789.

Bligh,E.G. and Dyer,W.J. (1959) Can. J. Physiol., 37, 911-917.

Burch,R.M., Jelsema,C. and Axelrod,J. (1988) J. Pharmacol. Exp. Ther., 244, 765-773.

Casey,P.J. and Gilman,A.G. (1988) J. Biol. Chem., 263, 2577-2580.

Church,J.G. and Buick,R.N. (1988) J. Biol. Chem., 263, 4242-4246.

Clark,M.A., Littlejohn,D., Conway,T.M., Mong,S., Steiner,S. and Crooke,S.T. (1986) J. Biol. Chem., 261, 10713-10718.

Daniel,T.O. and Ives,H.E. (1986) Biochem. Biophys. Res. Commun., 145, $111-117$.

Didsbury,J.R. and Synderman,R. (1987) FEBS Lett., 219, 259-263.

Didsbury,J.R., Ho,Y.-S. and Synderman,R. (1987) FEBS Lett., 211, $160-164$.

Downing,J.R., Rettenmier,C.W. and Sherr,C.J. (1988) Mol. Cell. Biol., 8, 1795- 1799 .

Folch,J., Lees,M. and Stanley,G.H.S. (1956) J. Biochem. Physiol., 37, 911-917.

Gilman,A.G. (1984) Cell, 36, 477-479.

Grinstein,S. and Rothstein,A. (1986) J. Membr. Physiol., 90, 1-12.

Guilbert,L.J. and Stanley,E.R. (1986) J. Biol. Chem., 261, 4024-4032.

Habenicht,A.J.R., Clomset,J.A., King,W.C., Nist,C., Michell,C.D. and Ross, R. (1981) J. Biol. Chem., 256, 12329-12335.

Hamilton,J.A., Vairo,G. and Lingelbach,S.R. (1988) J. Cell. Physiol., 134, 405-412.

He,Y., Hewlett,E., Temeless,D. and Quesenberry,P. (1988) Blood, 71, $1187-1195$

Hesketh,T.R., Moore,J.P., Morris,J.D., Taylor,M.V., Roger,J., Smith,G.A. and Metcalfe,J.C. (1985) Nature, 313, $481-484$.

Hidaka,H., Inagaki,M., Kawamoto,S. and Sasaki, Y. (1984) Biochemistry, 23, 5036-5041.

Horiguchi,J., Warren,M.K. and Kufe,D. (1987) Blood, 69, 1259-1261.

Horiguchi,J., Sariban,E., and Kufe,D. (1988) Mol. Cell. Biol., 8, $3951-3954$.

Imamura,K. and Kufe,D. (1988) J. Biol. Chem., 263, 14093-14098.

Irving,H.R. and Exton,J.H. (1987) J. Biol. Chem., 262, 3440-3443.

Jackowski,S., Rettenmier,C.W., Sherr,C.J. and Rock,C.O. (1986) J. Biol. Chem., 261, 4978-4985.

Jackowski,S., Rettenmier,C.W. and Rock,C.O. (1990) J. Biol. Chem., 265, 6611-6616.

Johnson,R.M., Connelly,P.A., Sisk,R.B., Pobiner,B.F., Hewlett,E.L. and Garrison,J.C. (1986) Proc. Natl. Acad. Sci. USA, 83, 2032-2036.

Kikuchi,A., Kozawa,O., Kaibuchi,K., Katada,T., Ui,M. and Takai,Y. (1986) J. Biol. Chem., 261, 11558-11562.

L'Allemain,G. and Pouyssegur,J. (1986) FEBS Lett., 97, 344-348.

Macara,I.G. (1985) Am. J. Physiol., 17, C3-C11.

Margolis,B., Rhee,S.G., Felder,S., Mervic,M., Lyall,R., Levitzki,A., Ullrich,A., Zilberstein,A. and Schlessinger,J. (1989) Cell, 57, $1101-1107$.

Meisenhelder,J., Suh,P.-G., Rhee,S.G. and Hunter,T. (1989) Cell, 57, 1109-1122.

Morrison,D.K., Browning,P.J., White,M.F. and Roberts,T.M. (1988) Mol. Cell. Biol., 8, 176-185.

Neer,E.J. and Clapham,D.E. (1988) Nature, 333, $129-134$.

Nienhuis,A.W., Bunn,H.F., Turner,P.H., Gopal Venkat,V., Nash,W.G., O'Brien,S.J. and Sherr,C.J. (1985) Cell, 42, 421-428.

Rosoff,P.M., Savage,N. and Dinarello,C.A. (1988) Cell, 54, 73-81.

Rothenberg,P.L. and Kahn,C.R. (1988) J. Biol. Chem., 263, 15546-15552.

Rozengurt,E. (1986) Science, 234, 161-166.

Sariban,E., Mitchell,T. and Kufe,D. (1985) Nature, 316, 64-66.

Sengupta,A., Liu,W.-K., Yeung,Y.G., Yeung,D.C.Y., Frackelton,Jr,A.R. and Stanley,E.R. (1988) Proc. Natl. Acad. Sci. USA, 85, 8062-8066.

Sherr,C.J., Rettenmier,C.W., Sacca,R., Roussel,M.F., Look,A.T. and Stanley,E.R. (1985) Cell, 41, 665-676.

Skipski,V.P., Peterson,R.F. and Barklay,M. (1964) Biochem. J., 90, 374-378.

Stanley,E.R., Guilbert,L.J., Tushinski,R.J. and Bartelemez,S.H. (1983)

J. Cell Biochem., 21, 151-159.
Stone,R.M., Spriggs,D.R. and Kufe,D.W. (1988) Blood, 72, 739-744.

Tamaoki,T., Nomoto,H., Takahashi,I., Kato,Y., Motomoto,M. and

Tomita,F. (1987) Biochem. Biophys. Res. Commun., 135, 397-402.

Teitelbaum,I. (1990) J. Biol. Chem., 265, 4218-4222.

Tushinski,R.J. and Stanley,E.R. (1983) J. Cell Physiol., 116, 67-75.

Tushinski,R.J. and Stanley,E.R. (1985) J. Cell. Physiol., 122, 221-228.

Vairo,G. and Hamilton,J.A. (1988) J. Cell. Physiol., 134, 13-24.

Vairo,G., Argyriou,S., Bordun,A.-M., Whitty,G. and Hamilton,J.A. (1990) J. Biol. Chem., 265, 2692-2701.

Vara,F., Schneider,J.A. and Rozengurt,E. (1984) Proc. Natl. Acad. Sci. $U S A, 82,2384-2388$.

Vara,F. and Rozengurt,E. (1985) Biochem. Biophys. Res. Commun., 130, 646-653.

Vegesna,R.V., Wu,H.-L., Mong,S., and Crooke,S.T. (1988) Mol. Pharmacol., 33, 537-542.

Wahl,M.I., Olashan,N.E., Nishibe,S., Rhee,S.G., Pledger,W.J. and Carpenter,G. (1989) Mol. Cell. Biol., 9, 2934-2943.

Wakamiya,N., Horiguchi,J. and Kufe,D. (1987) Leukemia, 1, 518-520.

Warden,C.H. and Friedkin,M. (1985) J. Biol. Chem., 260, 6006-6011.

Whetton,A.D., Monk,P.N., Consalvey,S.D. and Downes,C.P. (1986) EMBO J., 5, $3281-3286$.

Whetton,A.D., Monk,P.N., Consalvey,S.D., Huang,S.J., Dexter,T.M. and Downes,C.P. (1988) Proc. Natl. Acad. Sci. USA, 85, 3284-3288.

Yarden,Y. and Ullrich,A. (1988) Biochemistry, 27, 3113-3119.

Yavin,E. (1976) J. Biol. Chem., 251, 1392-1397.

Received on June 20, 1989; revised on August 17, 1989 